\title{
Toxoplasma gondii seroprevalence in extensively farmed wild boars (Sus scrofa) in Denmark
}

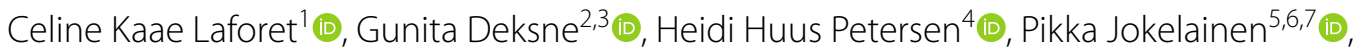 \\ Maria Vang Johansen ${ }^{1}$ (D) and Brian Lassen ${ }^{1 *}$ (D)
}

\begin{abstract}
Toxoplasma gondii is a zoonotic parasite of worldwide importance. In this study, we estimated T. gondii seroprevalence in extensively farmed wild boars in Denmark, where little is known about T. gondii in animal hosts. Our study focused on wild boars because they are considered good indicator species for the presence of T. gondii, and wild boar meat is used for human consumption. Serum samples from 101 wild boars collected in 2016-2018 from five different locations from the continental part of Denmark, Jutland, were screened for anti-T. gondii antibodies. The samples were analysed using a commercial indirect enzyme-linked immunosorbent assay (ELISA). Samples from 28 (27.7\%) of the 101 wild boars tested positive with the ELISA. The odds for a wild boar to test seropositive were higher if it was sampled during the hunting season 2017-2018 than during 2016-2017 and if it was reported to be at least 1 year old than if it was younger (logistic regression model with the two variables: odds ratios 17.5 and 3.9, respectively). A substantial proportion of the investigated extensively farmed wild boars had been exposed to T. gondii. Moreover, the parasite appeared widespread, at least in the continental part of Denmark, Jutland, as seropositive wild boars were found from all five sampled locations. Assuming seropositivity indicates hosting viable parasites, consumption of undercooked wild boar meat from Denmark is a potential source of T. gondii infections to other hosts, including humans.
\end{abstract}

Keywords: Serology, Sus scrofa, Toxoplasma gondii, Wild boar, Zoonosis

\section{Findings}

The zoonotic protozoan parasite Toxoplasma gondii has a wide host range [1], including wild boars (Sus scrofa). Wild boars are considered good indicator hosts for the presence of $T$. gondii, and seroepidemiological studies have shown that farmed and free-ranging wild boars are commonly exposed to T. gondii in several countries $[2,3]$. Toxoplasma gondii infections of wild boars are typically subclinical, but clinical congenital toxoplasmosis has been reported [4]. More importantly, T. gondii infections of wild boars are of public health importance, because wild boars are farmed and hunted for human

\footnotetext{
*Correspondence: brian.lassen@gmail.com

${ }^{1}$ Department of Veterinary and Animal Sciences, University

of Copenhagen, Grønnegårdsvej 15, 1870 Frederiksberg C, Denmark

Full list of author information is available at the end of the article
}

consumption. Thus, undercooked tissues of wild boars that are infected with T. gondii may serve as infection source to humans. Among zoonotic foodborne parasites of greatest concern, T. gondii has been ranked 4th globally [5] and 2nd in Europe [6].

In Denmark, free-ranging wild boars are currently not welcomed. However, wild boars are extensively farmed in fenced areas where hunters regulate the population during hunting seasons. In the Danish Central Livestock Register, altogether 63 fenced areas are listed for keeping wild boars [7]. Toxoplasma gondii has recently gained attention on the human medical side in Denmark $[8,9]$. However, major knowledge gaps in the local epidemiology of this zoonotic parasite exist, as only few studies have focused on animal hosts [10]. Literature searches 
identified no previous studies on T. gondii in wild boars from Denmark.

The aim of this seroepidemiological study was to estimate $T$. gondii seroprevalence in extensively farmed wild boars in Denmark. The sampling was a convenience sample from five separate locations in the continental part of Denmark, Jutland. The wild boars lived in fenced outdoor areas where they received supplementary feeding. The sampling took place during two hunting seasons, from October 2016 to January 2017 and from October 2017 to January 2018. The blood samples were collected post mortem from legally hunted wild boars. Sera were separated by centrifugation and stored at $-21^{\circ} \mathrm{C}$ until the analysis.

The samples were analysed for antibodies against $T$. gondii using a commercial indirect enzyme-linked immunosorbent assay (ELISA; ELISA ID Screen Toxoplasmosis Indirect Multi-species, IDvet, Grabels, France), following the instructions of the manufacturer. The samples and the controls provided in the kit were analysed in duplicate. The optical density (OD) was read at $450 \mathrm{~nm}$. Results were evaluated by calculating the $\mathrm{S} / \mathrm{P} \%$ (sample/positive percentage $)=($ mean OD of sample - mean OD of negative control)/(mean OD of positive control - mean OD of negative control) $\times 100$. Samples with $\mathrm{S} / \mathrm{P} \% \leq 40 \%$ were considered negative, $40-50 \%$ doubtful, and $\geq 50 \%$ positive.

The outcome was dichotomised: wild boars that tested positive with the ELISA were considered seropositive; others were considered seronegative. The animal-level variables evaluated were 'season' (sample collected in 2016-2017 vs. 2017-2018), 'age group' (reported to be $<1$ year old vs. at least 1 year old) and 'sex' (female vs. male). The locations were coded $1-5$, and evaluated as dummy variables 'location'. Each variable was initially analysed alone (crude, univariable analysis). Secondly, all four variables were included in a logistic regression model, followed by step-wise removal until only significant variables and confounders were left.

OpenEpi [11] was used to evaluate sample size, to calculate confidence intervals (CI) for the proportions (Mid-P Exact), and to compare proportions (e.g. the seroprevalence in the two age groups; our seroprevalence estimate vs. estimates reported in other studies) using two-by-two tables (2-tailed $P$ value, Mid-P Exact). Logistic regression analyses were performed using Stata 13.1 (StataCorp, College Station, TX, USA). P-values (Mid-P exact; those calculated by Stata) $<0.05$ were considered significant.

The minimum needed sample size for estimating the seroprevalence was calculated to be 85-91 animals, based on the seroprevalence estimates of $33 \%$ and $50 \%$ from Finland and Sweden, respectively [12, 13], a $80 \%$ confidence level, and a population size of 200 (estimated annual wild boar hunting bag in Denmark). The sample size available for the study was 101 wild boars, 38 (37.6\%) of which were reported to be younger than 1 year old. Altogether 53 (52.5\%) were female and 47 (46.5\%) were male (Table 1). Age group was not reported for two wild boars, and sex was not reported for one wild boar.

Samples from 28 (27.7\%, 95\% CI 19.7-37.1) of the 101 wild boars tested positive with the ELISA. The manufacturer's cut-off values for considering the test as validated were met. Four wild boars tested doubtful (considered seronegative in the interpretation of results).

The seroprevalence estimate was lower in 2016-2017 than in 2017-2018 (Table 1, P-value $<0.001$ ). Based on univariable model, the odds to test seropositive were 14.9 (95\% CI 3.3-67.5) times higher in wild boars sampled in 2017-2018 than in those sampled in 2016-2017. The seroprevalence estimate in wild boars reported to be at least 1 year old was significantly higher than the estimate in reportedly younger wild boars (Table 1 , P-value $<0.05$ ). Based on univariable model, the odds to test seropositive were 3.0 (95\% CI 1.1-8.3) times higher in wild boars reported to be at least 1 year old than in reportedly younger wild boars. The seroprevalence estimate was $35.8 \%$ in females and $19.1 \%$ in males; this difference was not statistically significant. Several seropositive wild boars were found from all five locations (Table 1). The variables 'sex' and 'location' were omitted from the multivariable model as non-significant; the final model used 99 observations (those with data available for the variables) and contained the variables 'season' and 'age group' (odds ratio 17.5, 95\% CI 3.7-81.6, and odds ratio 3.9, 95\% CI 1.3-11.8, respectively).

This study is the first to report seroprevalence of $T$. gondii in wild boars in Denmark and documents a wide exposure among extensively farmed wild boars. The results add to the scarce knowledge on $T$. gondii in its animal hosts in Denmark.

The seroprevalence estimate of $27.7 \%$ was obtained using an ELISA kit intended for samples from several host species, including pigs. However, the sensitivity and specificity are not reported. Partly because the seroprevalence estimate for 2016-2017 was unexpectedly low (Table 1), we tested the 41 samples from that hunting season also using a commercial modified direct agglutination test (DAT; Toxo-Screen DA, bioMérieux, Marcyl'Étoile, France; samples diluted 1:40). Samples from four (9.8\%, 95\% CI 3.2-21.9) of the 41 wild boars tested positive with the DAT. The DAT-results from each sample and its duplicate were consistent. The four DAT-positive samples comprised both ELISA-positive samples, one sample that tested negative with the ELISA, and one sample that tested doubtful with the ELISA. The DAT has 
Table 1 Toxoplasma gondii seroprevalence in extensively farmed wild boars (Sus scrofa) in Denmark, by hunting season, age group, sex, and location

\begin{tabular}{|c|c|c|c|c|}
\hline & $\begin{array}{l}\text { No. } \\
\text { of animals }\end{array}$ & $\begin{array}{l}\text { No. } \\
\text { of seropositive } \\
\text { animals }\end{array}$ & $\begin{array}{l}\text { Seroprevalence } \\
(\%)\end{array}$ & $\begin{array}{l}95 \% \\
\text { confidence } \\
\text { interval }\end{array}$ \\
\hline \multicolumn{5}{|l|}{ Season } \\
\hline 2016-2017 & 41 & 2 & 4.9 & $0.8-15.2$ \\
\hline 2017-2018 & 60 & 26 & 43.3 & $31.3-56.0$ \\
\hline \multicolumn{5}{|c|}{ Age group (years) } \\
\hline$<1$ & 38 & 6 & 15.8 & $6.7-30.0$ \\
\hline$\geq 1$ & 61 & 22 & 36.1 & $24.8-48.6$ \\
\hline \multicolumn{5}{|l|}{ Sex } \\
\hline Female & 53 & 19 & 35.8 & $23.8-49.4$ \\
\hline Male & 47 & 9 & 19.1 & $9.8-32.2$ \\
\hline \multicolumn{5}{|l|}{ Location } \\
\hline Location 1 & 32 & 7 & 21.9 & $10.1-38.6$ \\
\hline Location 2 & 14 & 6 & 42.9 & $19.6-68.9$ \\
\hline Location 3 & 10 & 4 & 40.0 & $14.2-70.9$ \\
\hline Location 4 & 26 & 7 & 26.9 & $12.6-46.1$ \\
\hline Location 5 & 19 & 4 & 21.1 & $7.1-43.3$ \\
\hline Total & 101 & 28 & 27.7 & $19.7-37.1$ \\
\hline
\end{tabular}

Age group was unknown for two wild boars, sex was unknown for one wild boar

no host-species-specific reagents and it has been widely used for both domestic pigs and wild boars. DAT detects only specific immunoglobulin $G$ antibodies, because possible immunoglobulin $M$ antibodies are denatured by 2 -mercaptoethanol, and very recent infections where these antibodies are not yet formed are missed [2]. The DAT-titer of 40, which we used as cut-off to define seropositivity can be considered high [1, 14], and thus even the DAT-based estimate (which for these 41 samples is the same as if the two methods would have been used in parallel, which increases sensitivity) may underestimate the infection prevalence. Infective $T$. gondii has been isolated from wild boars with lower DAT-titers than our cut-off for seropositivity $[14,15]$. Further, we ruled out the possibility that the difference could have been due to clustering by location. Three of the five locations were sampled during both hunting seasons, and the results from this subgroup were similar to the overall results ( $\mathrm{n}=77$, ELISA-seroprevalence $2 / 41,4.9 \%, 95 \%$ CI $0.8-$ 15.2 in $2016-2017$ and $16 / 36,44.4 \%, 95 \%$ CI $29.0-60.8$ in 2017-2018, P-value <0.001, univariable OR 15.6, 95\% CI 3.3-74.7). Monitoring the situation over several years could be useful to investigate the annual variation and, in particular, whether our study with two sampling time points identified a true increase in seroprevalence.

The seroprevalence was significantly higher in the wild boars reported to be at least 1 year old than in those reported to be younger. This result is in line with the result from free-ranging wild boars from Sweden [13], and indicates that the infections are acquired: older wild boars have had longer time to encounter the parasite. Whether the antibodies persist lifelong in wild boars has been questioned [16]. Hence, negative results, in particular in older wild boars, may not rule out earlier exposure, and the estimate in the older age group may be an underestimation of the proportion exposed. Regardless, it is also noteworthy that the seroprevalence estimate in wild boars reported to be below 1 year of age was already relatively high, $15.8 \%$, as this indicates substantial infection pressure. In the Nordic-Baltic region, Estonia is a country where T. gondii infection pressure appears particularly high, and there, no statistically significant difference in seroprevalence between age groups was observed in free-ranging wild boars $(22.4 \%$ in younger age group and $27.6 \%$ in older age group) [17].

In this study, T. gondii seroprevalence was 1.9 times higher in females than in males, but the difference was not statistically significant. In farmed wild boars in Finland, the difference was similar, 1.9-fold and significant, and females had significantly higher odds to test seropositive than males also based on the results of a multivariable random effects logistic regression model [12]. Whether and why female wild boars could be more exposed to the parasite on the farms than male wild boars would merit further studies.

Seropositive wild boars were detected from all five sampled locations in the continental part of Denmark, Jutland, but the results may not represent the situation in other parts of Denmark nor the environment outside the fenced areas.

A recent systematic review and meta-analysis on T. gondii in wild boars estimated the seroprevalence to be $23 \%$ globally and $26 \%$ in Europe [3]. Our overall estimate $(27.7 \%)$ was thus of the expected magnitude, and did not differ significantly from those reported for farmed wild boars in Finland (33.0\%) [12] and Latvia (20.3\%) [18] nor from those reported for free-ranging wild boars in Latvia (35.1\%) [18], Estonia (24.0\%) [17], and Sweden (28.6\%) [19], but it was lower (P-value $<0.001)$ than an earlier estimate for freeranging wild boars in Sweden (49.5\%) [13]. It should be emphasized that the results of different studies are not directly comparable due to different farm management and game management, different sampling strategies, and different methods applied, but they illustrate that the parasite is common and endemic in the Nordic-Baltic region.

Because wild boars are considered good indicators for $T$. gondii, our results suggest the zoonotic parasite is common and widespread in the extensive wild boar farming environment in the continental part of 
Denmark, Jutland. Because T. gondii seropositivity indicates exposure, chronic infection, and carrying infective parasites in the tissues, eating undercooked meat from wild boars from Denmark may present $T$. gondii infection risk to other hosts, including humans.

\section{Abbreviations}

Cl: confidence interval; DAT: modified direct agglutination test; ELISA: enzymelinked immunosorbent assay; OD: optical density; S/P: sample/positive.

\section{Authors' contributions}

BL and PJ presented the idea of the study. CKL, BL and MVJ designed the study. HHP coordinated and performed the sampling. CKL, GD and HHP performed the serology. CKL, BL and PJ analysed the data. CKL, PJ and BL drafted the manuscript. All authors read and approved the final manuscript.

\section{Author details}

${ }^{1}$ Department of Veterinary and Animal Sciences, University of Copenhagen, Grønnegårdsvej 15, 1870 Frederiksberg C, Denmark. ${ }^{2}$ Institute of Food Safety, Animal Health and Environment, "BIOR", Lejupes Str. 3, Riga, Latvia. ${ }^{3}$ Faculty of Biology, University of Latvia, Jelgavas Str. 1, Riga, Latvia. ${ }^{4}$ Section for Diagnostics and Scientific Advice, National Veterinary Institute, Technical University of Denmark, Kemitorvet, 2800 Kgs. Lyngby, Denmark. ${ }^{5}$ Laboratory of Parasitology, Department of Bacteria, Parasites \& Fungi, Infectious Disease Preparedness, Statens Serum Institut, Artillerivej 5, 2300 Copenhagen S, Denmark. ${ }^{6}$ Faculty of Veterinary Medicine, University of Helsinki, P.O. Box 66, 00014 Helsinki, Finland. ${ }^{7}$ Institute of Veterinary Medicine and Animal Science, Estonian University of Life Sciences, Kreutzwaldi 62, 51014 Tartu, Estonia.

\section{Acknowledgements}

We thank the locations for their collaboration and the staff of Institute of Food safety, Animal health and Environment "BIOR" for kindly hosting CKL.

\section{Competing interests}

The authors declare that they have no competing interests.

\section{Availability of data and materials}

The datasets used and analysed during the current study are available from the corresponding author on reasonable request.

\section{Ethics approval and consent to participate}

This study did not require official or institutional ethical approval. No animals were hunted for the purpose of this study. Certified hunters shot the wild boars during hunting seasons, and the samples were collected for a national surveillance program of infectious diseases in wild boars. The samples were collected post mortem. The data were handled confidentially.

\section{Funding}

Danish Veterinary and Food Administration funded the collection of samples and analysis of some of the samples. Funding for materials was received from Foreningen af Veterinærvidenskabelige Medarbejderes Fond. The study was supported by the CYSTINET Cost Action TD1302 in the frame of a Short Term Scientific Mission.

\section{Publisher's Note}

Springer Nature remains neutral with regard to jurisdictional claims in published maps and institutional affiliations.
Received: 25 September 2018 Accepted: 7 January 2019

Published online: 15 January 2019

\section{References}

1. Dubey JP. Toxoplasmosis of animals and humans. 2nd ed. Boca Raton: CRC Press; 2010.

2. Dubey JP. Toxoplasmosis in pigs - the last 20 years. Vet Parasitol. 2009;164:89-103.

3. Rostami A, Riahi SM, Fakhri Y, Saber V, Hanifehpour H, Valizadeh S, et al. The global seroprevalence of Toxoplasma gondii among wild boars: a systematic review and meta-analysis. Vet Parasitol. 2017;244:12-20.

4. Calero-Bernal R, Gómez-Gordo L, Saugar JM, Frontera E, Pérez-Martín JE, Reina D. Congenital toxoplasmosis in wild boar (Sus scrofa) and identification of the Toxoplasma gondii types involved. J Wildl Dis. 2013;49:1019-23.

5. FAO/WHO, Food and Agriculture Organization/World Health Organization. Multicriteria-based ranking for risk management of food-borne parasites. Microbiol Risk Assess. 2014;23:16.

6. Bouwknegt M, Devleesschauwer B, Graham H, Robertson LJ, van der Giessen JW, The EURO-FBP Workshop Participants. Prioritisation of foodborne parasites in Europe, 2016. Euro Surveill. 2018;23:17-00161.

7. Danish Central Livestock Register. 2018. https://chr.fvst.dk/chri/faces/ frontpage? adf.ctrl-state=p338km7td_3. Accessed 5 Jan 2019.

8. Nissen J, Jokelainen P, Stensvold CR, Trevisan C, Fuchs J, Burgdorf KS, et al. The disease burden of congenital toxoplasmosis in Denmark, 2014. PLoS ONE. 2017;12:e0178282.

9. Jokelainen P, Murat JB, Nielsen HV. Direct genetic characterization of Toxoplasma gondii from clinical samples from Denmark: not only genotypes II and III. Eur J Clin Microbiol Infect Dis. 2018;37:579-86.

10. Kofoed KG, Vorslund-Kiær M, Nielsen HV, Alban L, Johansen MV. Seroprevalence of Toxoplasma gondii in Danish pigs. Vet Parasitol Reg Stud Rep. 2017:10:136-8.

11. Dean AG, Sullivan KM, Soe MM. 2015. OpenEpi: open source epidemiologic statistics for public health. Version 3.01. http://www.openepi.com. Accessed 5 Jan 2019

12. Jokelainen $P$, Näreaho $A$, Hälli $O$, Heinonen $M$, Sukura A. Farmed wild boars exposed to Toxoplasma gondii and Trichinella spp. Vet Parasitol. 2012:87:323-7.

13. Wallander C, Frössling J, Vågsholm I, Uggla A, Lundén A. Toxoplasma gondii seroprevalence in wild boars (Sus scrofa) in Sweden and evaluation of ELISA test performance. Epidemiol Infect. 2015;143:1913-21.

14. Richomme C, Aubert D, Gilot-Fromont E, Ajzenberg D, Mercier A, Ducrot $C$, et al. Genetic characterization of Toxoplasma gondii from wild boar (Sus scrofa) in France. Vet Parasitol. 2009;164:296-300.

15. Puvanesuaran VR, Noordin R, Balakrishnan V. Genotyping of Toxoplasma gondii isolates from wild boars in Peninsular Malaysia. PLOS ONE. 2013;8:e61730

16. Opsteegh M, Swart A, Fonville M, Dekkers L, van der Giessen J. Agerelated Toxoplasma gondii seroprevalence in Dutch wild boar inconsistent with lifelong persistence of antibodies. PLOS ONE. 2011;6:e16240.

17. Jokelainen $P$, Velström $K$, Lassen B. Seroprevalence of Toxoplasma gondii in free-ranging wild boars hunted for human consumption in Estonia. Acta Vet Scand. 2015;57:42.

18. Deksne G, Kirjušina M. Seroprevalence of Toxoplasma gondii in domestic pigs (Sus scrofa domestica) and wild boars (Sus scrofa) in Latvia. J Parasitol. 2013:99(1):44-7.

19. Malmsten A, Magnusson U, Ruiz-Fons F, González-Barrio D, Dalin AM. A serologic survey of pathogens in wild boar (Sus scrofa) in Sweden. J Wildl Dis. 2018;54:229-37. 